\title{
Design and Implementation of Indoor Environmental Quality Monitoring System Based on ZigBee
}

\author{
X.M. Liu, F.F. Wang, Z.B. Zeng \\ Engineering Center of Dig ital Audio \& Video \\ Communication University of China \\ Beijing, China
}

\begin{abstract}
This paper designs a indoor environmental quality monitoring system, in which indoor environment parameters, such as temperature, humidity and PM2.5 density can be gathered, transmitted and displayed. Arduino, a open-source hardware and soft ware platforms, is used to access sensors. With the help of ZigBee, which is a new wireless communication protocol characterized by low-power, devices composed of Arduino and sensors are placed in different rooms so that the energy consumption can be effectively reduced. In addition, an specific transmission protocol is also be defined for this system. Finally sensor data are transmitted to the internal server and then a cloud platform via WiFi. The result shows that this system is stable and easy to build and change network.
\end{abstract}

Keywords-indoor environmental quality monitoring system; wireless sen sor networks; ZigBee; arduino

\section{INTRODUCTION}

In pace with the rapid development of society, the standard of human living has been improved greatly, health problems instead of basic necessities of life become the focus of attention. In the numerous factors influencing human health, effects caused by environment is particularly impressive. Because people have no place to hide if the environmental problems arise. At the same time, statistics show that about $80 \%$ of the time was spent in the indoor environment for most of human, thus the indoor environment has a direct impact on human health level[1]. Therefore, efficient monitoring of indoor environmental quality is important in both research value and practical significance. It is also the premise of home appliances controlling which could realize environment quality improvement.

Sensors play the role which could perceive environment parameters. Thus, how to construct a efficient and stable sensor network is the key problem of monitoring system design. Previously, sensor network exists in the form of wired network which is difficult to wiring mostly. Moreover, it is not flexible to make changes. In order to get rid of the shackles of cable, people began to try a Bluetooth or WiFi network. However, Bluetooth is not suitable for sensor network because it only supports point-to-point communication [2]. The transmission distance of Bluetooth is also too short. Although the transmission distance of $\mathrm{WiFi}$ is long more than enough, excessive power dissipation limits its application in sensor network [3]. The emerg ing ZigBee technology is very suitable for sensor network within the family whatever in the aspect of power dissipation or transmission distance. In addition,
ZigBee supports point to multipoint communication, the network capacity in a channel is up to 65536 [4].

\section{SYSTEM ARCHITECT URE}

The layered communication system including the perception layer, network layer and application layer is used to networking Internet of Things (IoT) usually. In this paper, the indoor environmental quality monitoring system is also designed according the hierarchical architecture of IoT. Th is system uses the equipment constructed of temperature/humidity sensor, PM2.5 sensor and Arduino micro-controller on the perception layer. The network layer consists of ZigBee and WiFi communication protocol. ZigBee is used as Wireless Sensor Networks (WSN) while WiFi plays a role to access the Internet. Finally, this system uses a internal server to store data and YeeLink, one of the earliest cloud platform in China to display. The system architecture is shown in fig. 1.

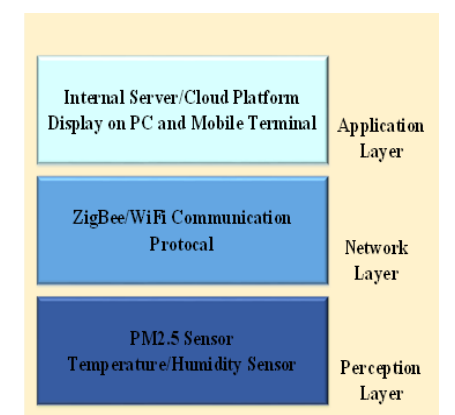

FIGURE I. SYSTEM ARCHITECTURE.

ZigBee protocol consists of the physical layer and the MAC layer defined by IEEE 802.15.4 protocol standard, the network layer and the application layer specified by Zig Bee Alliance. Fig. 2 gives the architecture of Zig Bee.

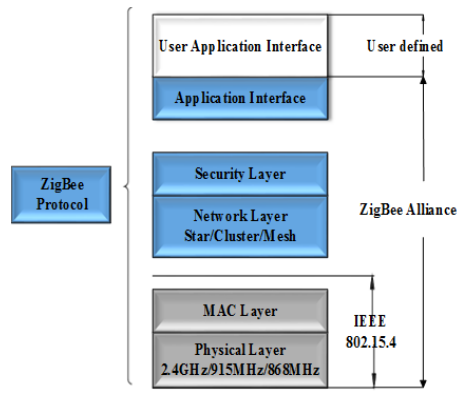

FIGURE II. ZIGBEE PROT OCOL DIAGRAM. 
This paper also defines a special transmission protocol for indoor environmental quality monitoring as the user application interface. In this protocol, the frame header consis ts of two bytes. The first byte is Room ID, and the last one is Physical Quantity Numbers. Table 1 shows the structure of the frame header. Room ID defines the location of the perception equipment. The first three bits of it indicate the type of a room. $0 \mathrm{~b} 000$ means this is a bedroom, while $0 \mathrm{~b} 001 \mathrm{a}$ washroom, $0 \mathrm{~b} 010$ a kitchen and $0 \mathrm{~b} 011$ a living room. There are also four types can be defined and added. The other 5 bits express the number of the room. Obviously, this system provides 32 rooms for each type, which can meet the needs of almost all family. The structure of Room ID is shown in table 2. Physical Quantity Numbers defines how many physical quantities are monitored by this system. It supports 256 different types of physical quantities without a specific type definition that means you can decide what kind of sensor should be placed in your room.

As is shown in table 3, the structure of Data defined in this protocol consists 6 bytes for each physical quantity monitored in this system. The first byte expresses the type of a physical quantity, while the second indicates data type. This system concludes 3 types of physical quantities. They are PM2.5 density, temperature and humidity, of course you can add your own new type of physical quantity. As for data type, it decides that this is a int type, float type or some others. After each Data Type byte, there are 4 bytes used to accommodate real data.

TABLE I. HEADER STRUCTURE.

\begin{tabular}{|c|c|}
\hline \multicolumn{2}{|c|}{ Header } \\
\hline Bytes 1 & Byte 2 \\
\hline Room ID & Physical Quantity Numbers \\
\hline
\end{tabular}

TABLE II. ROOM ID STRUCTURE.

\begin{tabular}{|l|c|c|c|c|c|c|c|}
\hline \multicolumn{7}{|c|}{ Room ID } \\
\hline bit 7 & bit 6 & bit 5 & bit 4 & bit 3 & bit 2 & bit 1 & bit 0 \\
\hline \multicolumn{3}{|c|}{ Room Type } & \multicolumn{5}{|c|}{ Room Number } \\
\hline
\end{tabular}

TABLE III. DATA STRUCTURE.

\begin{tabular}{|c|c|c|}
\hline \multicolumn{3}{|c|}{ Data } \\
\hline Byte 3 & Byte 4 & Bytes 5-8 \\
\hline Physical Quant ity Type & Data Type & Data1 \\
\hline Byte 9 & Byte 10 & Bytes 11-14 \\
\hline Physical Quant ity Type & Dat a Type & Dat a2 \\
\hline Byte 15 & Byte 16 & Bytes 17-20 \\
\hline Physical Quantity Type & Dat a Type & Dat a1 \\
\hline \multicolumn{3}{|c}{$\ldots}$. \\
\hline
\end{tabular}

ZigBee protocol concludes three different types of network topology structure, respectively, star, cluster tree and mesh topology structure. This system adopts the star topology, as in shown in fig. 3. In this system, a end node is placed in each room to collect and transmit data and a coordinator node provided to receive, convergence and deal with data uniformly. The coordinator traverse each end node in polling mode. It can also find new end nodes. That of course means you can move your end nodes to a more appropriate situation in the coverage area of coordinator signal. Zig Bee network has very strong fle xibility.

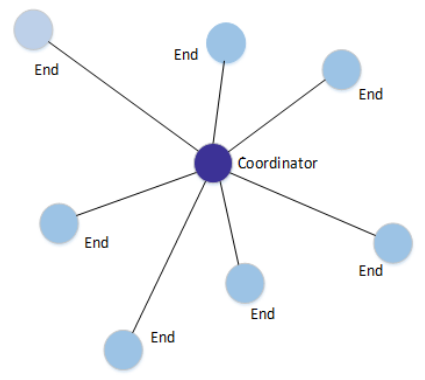

FIGURE III. ST AR TOPOLOGY.

\section{HARDWARE DESIGN}

\section{A. Selection of the Controller and the Protocol Chip}

This system uses Arduino UNO as the controller of the end nodes. Arduino UNO is an ATmega328-based development board, which has 14 ways of digital input/output (including 6 roads can be used as PWM output), 6 analog input and a $16 \mathrm{MHz}$ crystal. At the same time, Arduino Uno supports I2C and SPI serial bus. Therefore, it is very suitable a board as access platform for sensors. A WiDo development board based on ATmega32U4-based Arduino Leonardo and CC3000-based $\mathrm{WiFi}$ kernel is used to design the coordinator node. Among them, CC3000 is a low power WiFi function module of TI company designed for reducing the IOT threshold of house furnishings. WiDo, as a lightweight $\mathrm{WiFi}$ node, integrates a SD card for storing large amounts of data, is very suitable to play the role of a coordinator to accomplish data aggregation and connect Internet. In the selection of ZigBee protocol chip, this paper uses the XBee module made by DiGi company. The XBee module, with a great application layer interface, can greatly shorten the development cycle [5].

\section{B. Access Sensors}

To monitor temperature and humidity, this paper uses SILICON LABS's Si7021 digital te mperature/humid ity sensor. LM35 analog linear temperature sensor is also be used in this system. Si7021 sensor is a monolithic CMOS circuits integrated humidity and temperature sensors, ADC, signal processing module, calibration data and an I2C interface [6]. Its internal structure is shown in fig. 4.

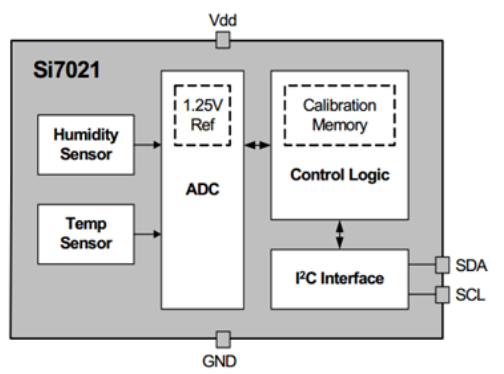

FIGURE IV. INTERNAL ST RUCT URE DIAGRAM OF SI7021.

In order to measure the temperature and humidity, Si7021 need an adaptation circuit to access Arduino controller. Fig. 5 shows the matching circuit of Si7021. Si7021 connects to Arduino via I2C interface. Se rial Data (SDA) and Serial Clock 
(SCL) of Si7021 should connect to a voltage between $1.9 \sim 3.5 \mathrm{~V}$ by a pull-up resistor. A small capacitance is used to filter the high frequency signal and provide a clean power for chip.

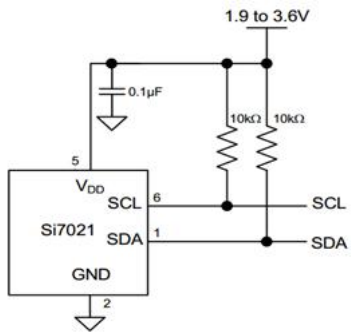

FIGURE V. MATCHING CIRCUIT OF SI7021.

When the sensor complete the measurement of humidity under the control of the controller, the measurement results can be converted into relative hu midity by the eqn. (1):

$$
R H(\%)=\frac{125 \times R H_{-} \text {Code }}{65536}-6
$$

Among them, RH (\%) humidity is corresponding relative humidity, $\mathrm{RH}$ _Code is the 16 bits of data returned through the I2C interface of Si7021.

Temperature measurement can also be converted into a temperature value that unit is Celsius via eqn. (2) :

$$
\text { Temperature }\left({ }^{\circ} \mathrm{C}\right)=\frac{175.72 \times \text { Temp_Code }}{65536}-46.85
$$

Among them, Temperature is the corresponding temperature value that unit is Celsius, Temp_Code is the 16 bits data from Si7021.

As for the monitoring of PM2.5, this paper uses the GP2Y1010AU0F optical dust sensor made by SHARP company. GP2Y1010AU0F arrangement an infrared light emitting diode (IRED) and a photosensitive diode diagonally, through which they can detect the reflected light of dust in the air [7], as shown in fig. 6. GP2Y1010AU0F can detect very fine particles effectively .

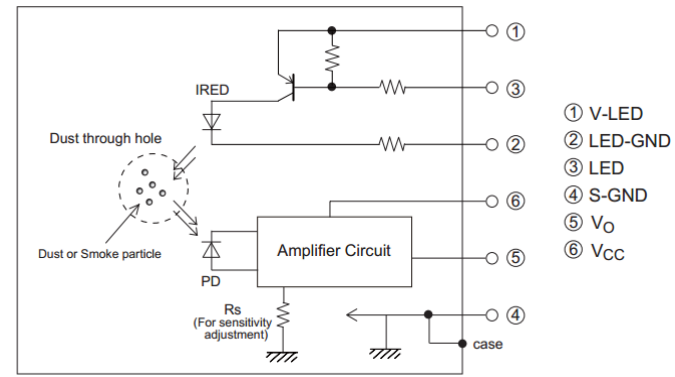

FIGURE VI

$$
\begin{aligned}
& \text { INTERNAL SCHEMATIC DIAGRAM OF } \\
& \text { GP2Y1010AU0F. }
\end{aligned}
$$

To measure the density of PM2.5 particulate matter in air, an adaptation circuit is also needed between the GP2Y1010AU0F sensor and Arduino. Fig. 7 gives a matching circuit of GP2Y1010AU0F. Vec is provided by Arduino. Resistor and capacitor besides build a RC low-pass filter, which has a good low-frequency performance and a strong interference.

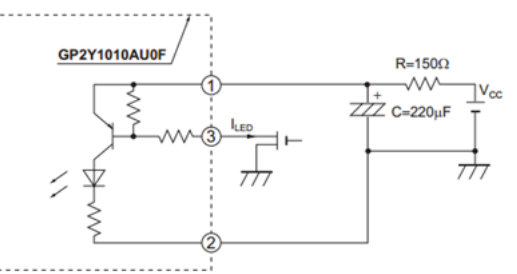

FIGURE VII.

MATCHING CIRCUIT OF GP2Y1010AU0F.

PM2.5 concentrations can be obtained by eqn. (3) and eqn. (4):

$$
\text { voCalculated }=\text { voMeasured } \times(5 / 1024)
$$

$$
\text { dustDensity }=0.17 \times \text { voCalculated }-0.1
$$

Among them, voCalcu lated represents the analog output of GP2Y1010AU0F, voMeasured is the digital value converted by $\mathrm{A} / \mathrm{DC}$, and dustDensity is the corresponding PM2.5 concentrations.

\section{Prototype}

Fig. 8 gives the prototype of this system. As is shown, there are four nodes in this paper, including a coordinator node and three end nodes. The end node numbered for No. 1 with a humid ity/temperature sensor and a PM2.5 sensor is placed in a living room, while the other two end nodes indexed for No. 2 and No.3 with a LM35 temperature sensor are putted in two different bedrooms. The coordinator node can be placed in any room you prefer, in fact, it is located at the living room in this paper.

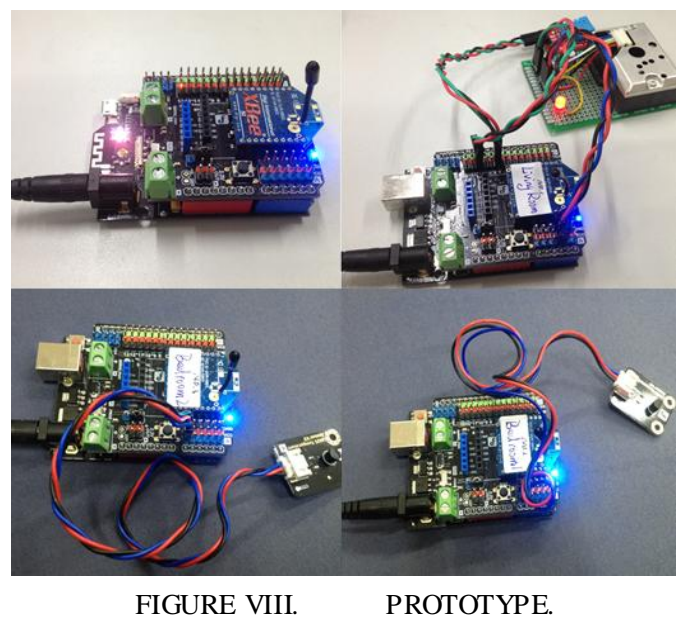

\section{SOFTWARE DESIGN}

\section{A. End Nodes}

A end node, as the sensing layer equipment, it needs accurate and efficient measurement of located air quality data. It also need to encapsulate the data so as to satisfy the 
agreement and transmits it to the coordinator after coordinator polling signal arrived. The program flow of end nodes is shown in fig. 9 (a).

\section{B. The Coordinator Node}

The coordinator node, as the center of the system, it needs to discover and recognition all of the end nodes around after be powered on. Then coordinator trans mits signals to each end node and receive feedback in polling mode. After got data comes from all of end nodes once, coordinator node should upload them to the cloud. Fig. 9 (b) gives the program flow of coordinator node.

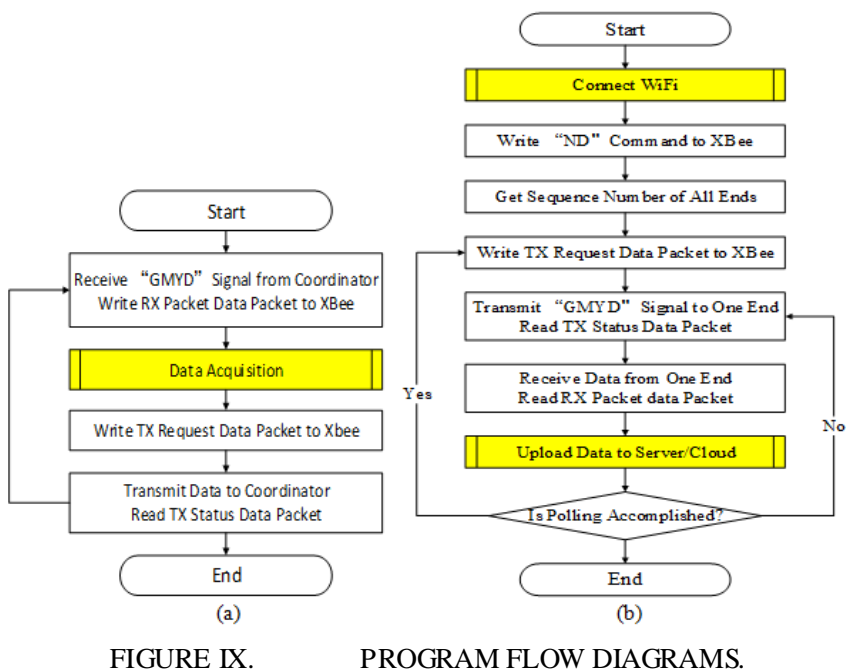

\section{Storage and Display}

This system build a internal server to receive data from the coordinator node. Data would also be upload to cloud platform, in this paper, YeeLink. Fig. 10 gives the data curve of one day in winter in Beijing of living room got from the storage server, while fig. 11 the real-time display of PM2.5 density on YeeLin k.
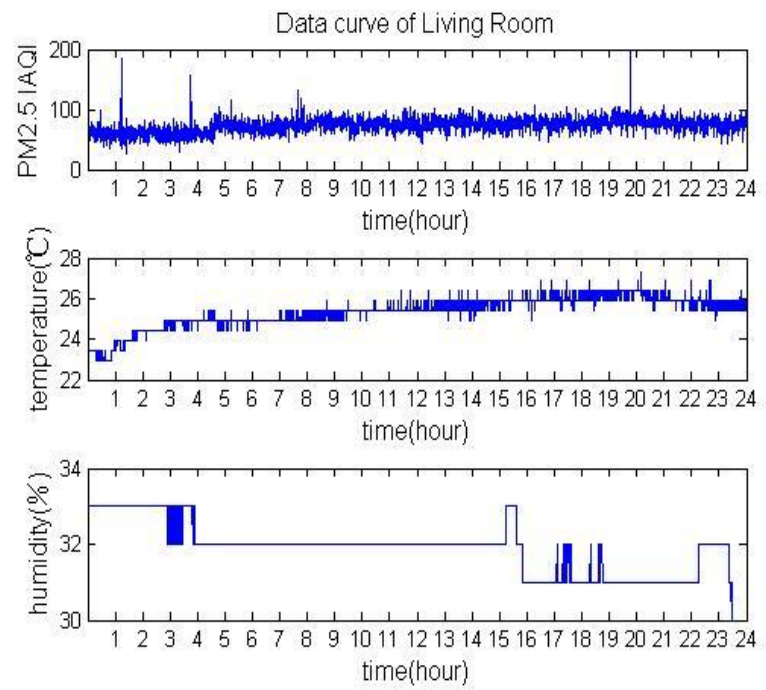

FIGURE X. DAT A CURVE OF LIVING ROOM.

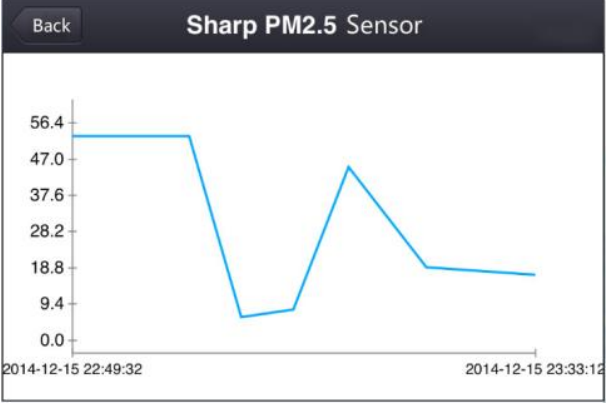

FIGURE XI.

REAL-TIME DISPLAY ON YEELINK (PM2.5 IAQI).

\section{CONCLUSIONS}

In this paper, an indoor environmental quality monitoring system was designed and implemented. This system uses Arduino as the controller to access sensors. Furthermore, XBee modules are used to establish a Zig Bee wireless network so that every end node can transmit the sensor data to the coordinator node. Finally, data are uploaded to the internal server and then YeeLink, by which data can be displayed on $\mathrm{PC}$ and Smart Phone. The results indicate that the system is effective and easy to add or delete nodes.

\section{REFERENCES}

[1] Bin Cui, Based on embeded household environment Intelligent surveillance system research (Master Thesis), Guangxi University of Technology, Liuzhou, Guangxi, China, 2011.

[2] Jin Woo Seo, Myung Suk Ryu, Kwang Suk Park and Do-Un Jeong, A home-based bedside monitoring system of ECG via bluetooth protocol. Biomedical Engineering, 2003 IEEE EMBS Asian-Pacific Conference on, pp. 30-31, 2003.

[3] Campoccia F., Sanseverino E.R., Zizzo,G., Analysis of the limitations of WiFi communications managed by the IEEE 802.11 protocol in data transmission in automated power distribution systems. Power Electronics Electrical Drives Automation and Motion (SPEEDAM), 2010 International Symposium on, pp. 352-357, 2010.

[4] Yi Xiaolin, Jia Zhigang, Chen Nanzhong, Zhu Wenjun and $\mathrm{Wu}$ Zhongning, The Research and Implementation of ZigBee Protocol-Based Internet of Things Embedded System. Information Engineering and Electronic Commerce (IEEC), 2010 2nd International Symposium on, pp $1-4,2010$.

[5] XBee®/XBee-PRO® RF Modules manual. Minnetonka: Digi International Inc, 2014.

[6] Si7021 I2C HUMIDITY AND TEMPERATURE SENSOR manual. Aust in: Silicon Laborat ories Inc, 2013.

[7] GP2Y1010AU0F Compact Optical Dust Sensor manual. Osaka: SHARP Corporation, 2006. 